\title{
Tafonomía de restos óseos y dentarios de micromamíferos recuperados en egagrópilas generadas por Megascops choliba (Strigiformes) en ambientes boscosos del centro de Argentina
}

\author{
Julián Mignino \\ Recibido 29 de septiembre 2020. Aceptado 17 de noviembre 2020
}

\begin{abstract}
RESUMEN
El estudio de micromamíferos recuperados en egagrópilas de rapaces es un insumo de utilidad para evaluar mecanismos de acumulación del registro arqueofaunístico, así como también para reforzar y fortalecer las inferencias paleoambientales. Con el objetivo de incrementar el conocimiento sobre agentes acumuladores en contextos arqueológicos y paleontológicos, este trabajo presenta el primer estudio tafonómico de restos óseos y dentarios recuperados en egagrópilas de la rapaz Megascops choliba en el Bosque Chaqueño Serrano del noroeste de la provincia de Córdoba, Argentina. Las muestras incluyen ocho especies de roedores sigmodontinos y una especie de marsupial Thylamyini. Se realizó un análisis tafonómico que evaluó el perfil taxonómico de las presas, la abundancia y proporciones de elementos anatómicos, patrones de fractura y evidencias de digestión. Los resultados de este análisis indicaron un alto porcentaje de abundancia relativa de partes esqueletales, con una preponderancia de elementos poscraneales por sobre los craneales y una leve pérdida de elementos distales respecto de los proximales. En relación con los patrones de fractura, si bien una elevada proporción de elementos craneales estaba fracturada, los elementos poscraneales generalmente se recuperaron completos. Por último, una alta proporción de dientes presentaba alteraciones por acción digestiva ligera. Estas observaciones indican que $M$. choliba puede ser incluida en las categorías de modificación moderada a intermedia.
\end{abstract}

Palabras clave: Micromamíferos; Neotafonomía; Zooarqueología; Análogos modernos; Strigiformes; Córdoba.

\section{Taphonomy of bone and tooth micromammal remains recovered from pellets generated by Megascops choliba (Strigiformes) in woody environments of central Argentina}

\begin{abstract}
The study of micromammals contained in raptor pellets is a useful tool to evaluate mechanisms of accumulation of the archaeofaunal record and to strengthen palaeoenvironmental inferences. With the goal of increasing knowledge about possible accumulating agents of micromammal remains in archaeological and paleontological contexts, this paper presents the first taphonomic study of bone and tooth remains recovered from Megascops choliba pellets from the Chaqueño Serrano Forrest in the northwest of the province of Córdoba, Argentina. The samples include eight species of sigmodontine rodents and one species of Thylamyini marsupial. The taxonomic profile of the prey, abundance and proportions of anatomical elements, breakage patterns and evidence of digestion were analyzed. The taphonomic evaluations indicated a high percentage of relative abundance of skeletal parts, with a preponderance of postcranial elements over the cranial, and a slight loss of distal elements with respect to the proximal. Regarding the fracture patterns, although a high proportion of cranial elements were detected fractured, the postcranial elements were generally recovered complete. Finally, a high proportion of teeth altered by light digestive action was detected. These observations reveal the inclusion of $M$. choliba in the categories of moderate to intermediate modification.
\end{abstract}

Keywords: Neo-taphonomy; Micromammals; Zooarchaeology; Modern analogous; Strigiforms; Córdoba.

Julián Mignino. Instituto de Antropología de Córdoba (IDACOR), Consejo de Investigaciones Científicas y Técnicas (CONICET), Museo de Antropología, Facultad de Filosofía y Humanidades (FFyH), Universidad Nacional de Córdoba (UNC), Córdoba, Argentina. Av. Hipólito Yrigoyen 174. E-mail: julianmignino@ffyh.unc.edu.ar

Intersecciones en Antropología 22(1), enero-junio: 69-81. 2021. ISSN-e 1850-373X https://doi.org/10.37176/iea.22.1.2021.590

Facultad de Ciencias Sociales - UNICEN - Argentina 


\section{INTRODUCCIÓN}

Los restos óseos y dentarios de microvertebrados, principalmente micromamíferos, son hallados con frecuencia en contextos arqueológicos y paleontológicos ubicados principalmente en sitios con reparos rocosos (Andrews, 1990). En relación con este aspecto, la tafonomía actualística se ha constituido como un campo esencial a la hora de evaluar las causas y agentes involucrados en la acumulación de restos óseos en sitios arqueológicos y paleontológicos. Este campo es también un insumo de utilidad para realizar inferencias de índole paleoambiental y paleobiológicas (Behrensmeyer y Kidwell, 1985; Andrews, 1990).

Desde esta perspectiva, en las últimas tres décadas se ha evidenciado un desarrollo considerable en aspectos teórico-metodológicos vinculados al estudio tafonómico de repertorios faunísticos en sitios con diversas características (e.g., Borrero, 1990; Montalvo, 2004; Izeta, 2005; Gutiérrez, 2009; Montalvo y Fernández, 2019; Scheifler et al., 2020).

Como consecuencia de lo mencionado, las investigaciones sobre restos de huesos y dientes de pequeños mamíferos $(\leq 1 \mathrm{~kg})$ se incrementaron en todo el Cono Sur sudamericano, con lo que se generó un acervo de datos y modelos tafonómicos regionales como análogos modernos de mayor precisión (Pardiñas, 1999; Frontini y Escosteguy, 2015; Montalvo y Fernández, 2019).

La metodología orientada a reconocer las causas y agentes involucrados en la génesis de agregados óseos y dentarios de pequeños mamíferos en distintos contextos estuvo guiada, durante mucho tiempo, por estudios actualistas de heces de mamíferos carnívoros y, principalmente, de egagrópilas de aves rapaces diurnas y nocturnas de Europa y África (Andrews, 1990; Fernández-Jalvo y Andrews, 1992).

Sin embargo, en los momentos en que Andrews (1990) diseñó la metodología para el estudio de pequeños mamíferos hallados en sitios arqueológicos y paleontológicos, no se incorporaron muestras de depredadores sudamericanos (Fernández et al., 2017). Esta no es una cuestión menor, ya que se ha demostrado que existen variaciones regionales en cuanto a los patrones tafonómicos generados por mamíferos carnívoros y aves rapaces en distintos contextos ecológicos (e.g., Montalvo y Fernández, 2019; Montalvo et al., 2020). Las causas de esta variabilidad se han interpretado como producto de distintos factores, que involucran el tamaño del depredador, el tamaño y disponibilidad de las presas, el grado de dureza o resistencia a las fracturas de sus elementos anatómicos y la estacionalidad en que esas muestras fueron depredadas (e.g., Andrews y Fernández-Jalvo, 2018; Montalvo et al., 2020).

En función de los factores mencionados, numerosos estudios han reportado tendencias tafonómicas generadas por distintos depredadores sobre sus presas, y utilizaron esta información como insumo tanto para la identificación de agentes y procesos intervinientes en la formación de conjuntos óseos como para la reconstrucción de paleoambientes (Montalvo y Fernández, 2019). La importancia de estos estudios radica en que, dadas determinadas características tanto etológicas y ecológicas de los pequeños vertebrados, como su alta sensibilidad a los cambios ambientales y estrechos requerimientos ecológicos, ha sido posible establecer el uso de micromamíferos como indicadores de fluctuaciones paleoambientales frente a la necesidad de conocer los escenarios en los cuales se produjeron las ocupaciones humanas y no humanas a lo largo del tiempo (e.g., Pardiñas, 1999; Teta et al., 2005; Fernández et al., 2012; Scheifler, Teta y Pardiñas, 2012; Mignino, Izeta y Cattáneo, 2018; Alcaráz, 2020; López y Chiavazza, 2020).

El estudio de la acción de depredadores como agentes acumuladores de micromamíferos provee herramientas de utilidad para conocer los procesos de formación de sitios. No obstante, aún existen muchos taxones de presas y depredadores por estudiar. Incluso aunque se los estudie, los datos generados no pueden ser abordados de manera global, dada la elevada variabilidad tafonómica generada por el mismo tipo de depredador en distintas regiones (Montalvo y Fernández, 2019).

El alicucu común (Megascops choliba) es uno de los búhos de tamaño pequeño (220-240 mm, 160 g) más comunes del neotrópico (Hilty y Brown, 1996). Su distribución comprende desde las islas de Centroamérica, en el norte, hasta el centro de Argentina y Uruguay, en el sur del continente americano; es decir que ocupa una variada cantidad de ambientes, entre los que se incluyen bosques abiertos, bordes de bosques, sabanas, áreas de cultivo y zonas urbanas. Frecuentemente, habita ambientes por debajo de los 1500 msnm (König y Weick, 2010). Esta rapaz posee hábitos crepusculares y nocturnos, nidifica en huecos de árboles o en nidos abandonados por otras aves, y las escasas investigaciones sobre su alimentación indican 
una dieta estacionalmente oportunista, que incluye sobre todo insectos (e.g., grillos, mariposas nocturnas, escarabajos, arañas) en estaciones cálidas, con un incremento de pequeños vertebrados hacia épocas frías (e.g., anuros, quirópteros y roedores) (del Hoyo, Elliot y Sargatal, 1999; Delgado, 2007).

A pesar de los conocimientos sobre los hábitos tróficos y la geonemia de $M$. choliba, los patrones tafonómicos que genera sobre sus presas no han sido explorados hasta el momento. Por ello, para incrementar el conocimiento sobre depredadores neotropicales y su rol como agentes acumuladores de restos de microvertebrados, en este trabajo se reportan los primeros resultados tafonómicos sobre restos óseos y dentarios de micromamíferos recuperados en egagrópilas generadas por $M$. choliba en el bosque Chaqueño Serrano del noroeste de la provincia de Córdoba. En este sentido, este trabajo busca conocer características diagnósticas y datos como análogo moderno, que permitan categorizar y evaluar su presencia como agente acumulador de restos óseos en asociaciones arqueológicas y paleontológicas.

\section{MATERIALES Y MÉTODOS}

Las muestras analizadas provienen de la localidad de Ongamira ( 900-1500 msnm), localizada al norte de las Sierras Chicas cordobesas $\left(30^{\circ} 46^{\prime} 28.94^{\prime \prime} \mathrm{S}\right.$, $\left.64^{\circ} 24^{\prime} 49.57^{\prime \prime} \mathrm{O}\right)$. El valle de Ongamira se encuentra ubicado en el departamento de Ischilín, noroeste de la provincia de Córdoba, en la región central de Argentina (Figura 1). Este valle se emplaza hacia el noroeste del cerro Uritorco, una de las elevaciones más importantes de la región, en el límite septentrional de las Sierras Chicas. Este incluye una formación cretácica en la cual se forman paredones y aleros donde ha sido posible registrar la presencia de diferentes ocupaciones humanas con economía de caza y recolección (Cattáneo e Izeta, 2016). Estos aleros consisten en concavidades de dimensiones muy variables, localizadas en los frentes rocosos, lo que resulta en espacios o recintos con techo, piso y una pared interior, que están abiertos al exterior $y$, por lo tanto, a la intemperie; es decir, proyecciones rocosas que sobresalen y pueden proveer abrigo (Zárate, 2016).

Por otra parte, se han detectado numerosos sitios arqueológicos a cielo abierto, vinculados a actividades de producción, consumo de alimentos y confección de cerámica (Robledo, 2019). En esos espacios, se destaca la abundante presencia de material lítico producto de la talla del cuarzo y otras materias primas, como así también numerosa cantidad de restos arqueobotánicos procedentes de distintas estructuras de combustión. A su vez, se han recuperado abundantes restos arqueofaunísticos asociados a distintos taxones, los cuales se encuentran representados, en mayor frecuencia, por camélidos y conjuntos de fauna de porte menor, tales como restos de valvas de caracoles terrestres (Cattáneo e Izeta, 2016).

Asociadas a las diversas materialidades producto de ocupaciones humanas, se identificó la ocurrencia de distintas especies de microvertebrados (aves y micromamíferos). En un estudio tafonómico llevado a cabo en uno de los sitios del área, se determinó que la presencia de esta clase de restos se vincularía principalmente con la acción acumuladora de aves rapaces (plausiblemente $T$. furcata), que habrían ocupado los aleros y depositado restos digeridos de sus presas en momentos en que los sitios fueron desocupados por los grupos humanos (Mignino et al., 2018).

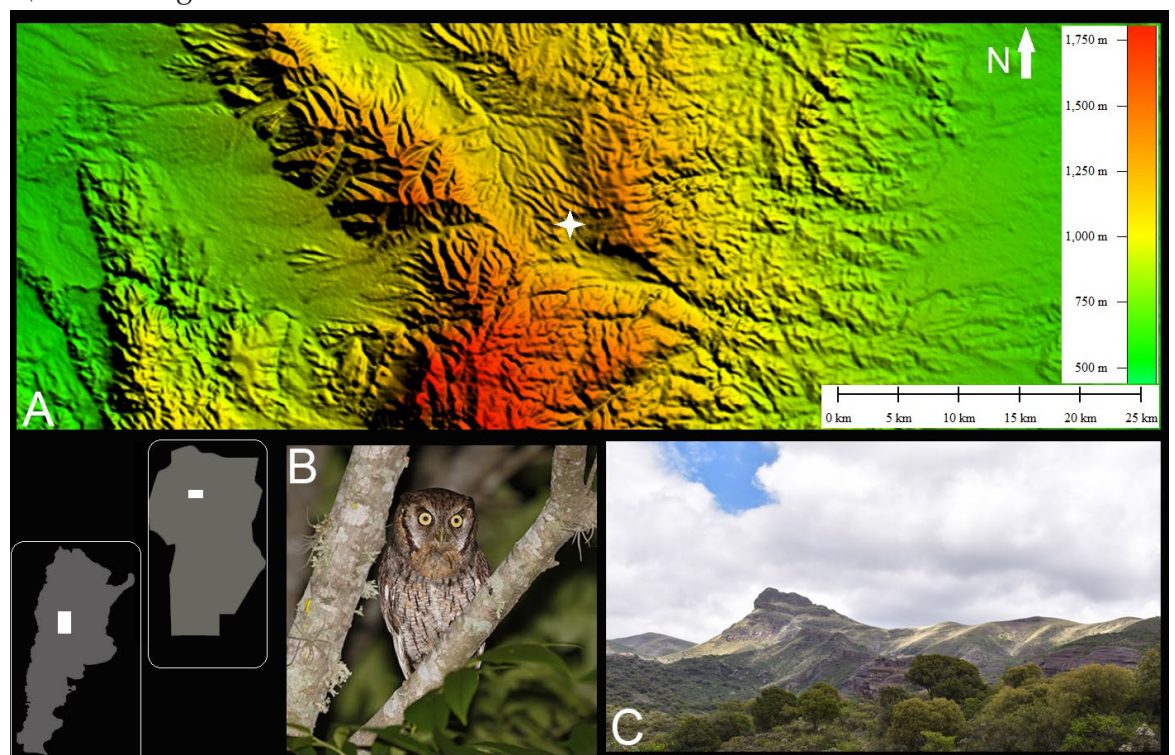

Figura 1. Sector bajo estudio $\left(30^{\circ} 46^{\prime} 28.94^{\prime \prime} \mathrm{S}, 64^{\circ} 24^{\prime} 49.57^{\prime \prime} \mathrm{O}\right)$. A. Ubicación general del sitio de muestreo de egagrópilas, en rojo se indica alero de procedencia. B. Ejemplar adulto de Megascops choliba. C. Vista general del valle. Se destacan áreas de bosque, roquedales y pastizales de altura. 
El clima en el valle se caracteriza como templado continental, con veranos calurosos y húmedos e inviernos fríos y secos. Entre los meses de junio y julio, ocurren nevadas ocasionales por sobre los $1000 \mathrm{msnm}$. Por otro lado, se registran rangos de temperatura entre los $9^{\circ} \mathrm{C}$ en los meses de junio-julio y $25{ }^{\circ} \mathrm{C}$ en los meses de enero-febrero. El régimen de precipitaciones es estacional, probablemente como réplica a las fluctuaciones estacionales de los anticiclones del Atlántico y el Pacífico sur; ya que presenta una variación entre $10 \mathrm{~mm}$ en estación seca (invierno) y 150 mm en estación cálida y épocas de lluvia (verano), con un patrón anual de precipitaciones de $870 \mathrm{~mm}$ (Piovano et al., 2009; Yanes et al., 2014). La vegetación se caracteriza por un estrato arbóreo dominado por especies típicas del bosque chaqueño como Lithraea molleoides (molle de beber), Schinopsis lorentzii (orco quebracho), Schinus areira (aguaribay) y Zanthoxylum coco (coco), entre otras (Robledo, 2019). También, se han registrado algunas especies de flora introducida, tales como Ligustrum lucidum (siempreverde).

Durante el 19 y 20 de septiembre de 2019 (estación fría y seca) se recolectaron 73 egagrópilas de $M$. choliba en una percha ubicada en un alero rocoso, 31 de las cuales contenían restos de micromamíferos. En las restantes, se registró material correspondiente a insectos, aves y anfibios, pero no fueron considerados en este trabajo. Se midió el largo y ancho de las egagrópilas con un calibre digital "Tesa", con un error de 0,01 mm.

Se separaron todos los restos y se los identificó taxonómica y anatómicamente con ejemplares comparativos y manuales de referencia (Fernández et al., 2011; Patton, Pardiñas y D'Elia, 2015; Mignino et al., 2018; Montalvo et al., 2020).

Las evaluaciones tafonómicas se realizaron utilizando una lupa binocular estereoscópica ("Motic" SMZ-140-N2GG, con una cámara de 3.0 megapíxeles) y se tomaron imágenes con un microscopio electrónico de barrido por emisión de campo (FESEM) (Zeiss FE-SEM Iigma, LaMARX, FAMAF, Universidad Nacional de Córdoba).

Se calculó el número de especímenes identificados por taxón (NISP), el número mínimo de individuos (MNI), sobre el elemento craneal homólogo mejor representado (mandíbula), y el número mínimo de elementos (MNE).

Para las evaluaciones sobre dieta, se estimó la biomasa de las presas. Este cálculo se expresa
$(\mathrm{Bi})=[(\mathrm{Spi} \mathrm{Ni}) / \Sigma(\mathrm{Spi} \mathrm{Ni})] \times 100$, donde Spi es el peso de la especie i y $\mathrm{Ni}$ es el número de individuos consumido de la especie i. En función de este cálculo, se estima la proporción en que cada taxón contribuyó al total de biomasa (peso) consumida (Marti, Bechard y Jaksic, 2007).

El estudio tafonómico se basó en el perfil taxonómico y de masas corporales de las presas, la abundancia relativa de elementos esqueléticos y los patrones de fractura sobre elementos craneales y poscraneales (fémures, tibias, húmeros, ulnas y radios). También se evaluaron modificaciones por corrosión digestiva sobre elementos diagnósticos (molares, incisivos, fémures y húmeros) (Fernández et al., 2017).

Siguiendo la propuesta metodológica de Andrews (1990) y de Fernandez-Jalvo y Andrews (1992), se calculó la proporción entre los elementos esqueléticos a partir de los índices: [(fémur + tibia + radio + ulna $) \times 8 /$ (mandíbula + maxilar + molares $) \times 5] \times 100$, [(húmero + fémur) / (mandíbula + maxilar) $] \times 100$ y [(tibia + radio) / (fémur + húmero)] $\times 100$.

Para evaluar la abundancia relativa de elementos esqueléticos, se calculó la siguiente fórmula:

$$
\mathrm{Ri}=\mathrm{MNEi} /(\mathrm{Ei} * \mathrm{MNI}) * 100
$$

Donde: $\mathrm{Ri}=$ abundancia relativa del elemento $\mathrm{i}$ MNEi = número de elementos $\mathrm{i}$ en la muestra $\mathrm{Ei}=$ frecuencia del elemento $\mathrm{i}$ en el esqueleto $\mathrm{MNI}=$ número mínimo de individuos

Para evaluar los patrones de fractura de elementos cráneo-mandibulares, se siguió la propuesta de Montalvo et al. (2020).

A fin de explorar las relaciones multivariadas entre muestras generadas por distintos depredadores y su posible aplicación para muestras fósiles y arqueológicas, se realizó un análisis de componentes principales (PCA). El análisis fue realizado con una matriz de datos que incluyó valores obtenidos en estudios tafonómicos actualísticos previos sobre restos de micromamíferos contenidos en egagrópilas y fecas de rapaces y mamíferos carnívoros. En la matriz, se incluyeron índices de abundancia relativa y representación de elementos esqueletales; porcentajes de fractura de elementos craneales y poscraneales; porcentajes de digestión en incisivos, molares y elementos del poscráneo. El análisis se realizó utilizando el software PAST -Paleontological Statistics- versión 4.03 (Hammer, Harper y Ryan, 2001). 


\section{RESULTADOS}

\section{Estructura taxonómica de comunidades presa consumidas por Megascops choliba}

Los resultados de identificaciones, representación y abundancia taxonómica de las muestras analizadas se presentan en la Tabla 1 y la Figura 2. En un total de 73 egagrópilas recolectadas, se identificaron micromamíferos en 31. Las medidas promedio de las egagrópilas analizadas fueron de $33,2 \times 22 \mathrm{~mm}$ y un peso seco promedio de 3,2 g. Se identificó un MNI total de 29. Entre ellos, Calomys musculinus (ratón maicero) es el sigmodontino de mayor representación ( $\% \mathrm{MNI}=31,03 \%)$, seguido por Graomys griseoflavus (pericote común) $(\% \mathrm{MNI}=17,24 \%)$ y Phyllotis xanthopygus (pericote panza gris) $(\% \mathrm{MNI}=17,24 \%)$. En menor proporción, se registraron Akodon polopi (ratón serrano) $(\% \mathrm{MNI}=10,34 \%)$, Calomys venustus (ratón cordobés) $(\% \mathrm{MNI}=6,99 \%)$, Oligoryzomys flavescens (ratón colilargo chico) $(\% \mathrm{MNI}=6,99 \%)$, Oxymycterus rufus (hocicudo rojizo) (\%MNI $=3,44 \%$ ) y Akodon dolores (ratón cordobés) $(\% \mathrm{MNI}=3,44 \%)$. Finalmente, se identificó la presencia del marsupial Thylamys pallidior (marmosa pálida) $(\% \mathrm{MNI}=3,44 \%)$.

\begin{tabular}{|l|c|c|c|c|}
\hline Taxón & $\begin{array}{c}\text { Promedio } \\
\text { masa } \\
\text { corporal } \\
\text { (g) }\end{array}$ & MNI & $\begin{array}{c}\% \\
\text { MNI }\end{array}$ & $\begin{array}{c}\% \\
\text { B }\end{array}$ \\
\hline RODENTIA \\
\hline Cricetidae \\
\hline Sigmodontinae \\
\hline Akodon polopi & 25 & 3 & 10,34 & 6,89 \\
\hline Akodon dolores & 50,5 & 1 & 3,44 & 4,63 \\
\hline Calomys musculinus & 17 & 9 & 31,07 & 14,05 \\
\hline Calomys venustus & 25 & 2 & 6,9 & 4,59 \\
\hline Phyllotis xanthopygus & 57 & 5 & 17,24 & 26,18 \\
\hline Oxymycterus rufus & 80 & 1 & 3,44 & 7,34 \\
\hline Graomys griseoflavus & 67,5 & 5 & 17,24 & 31 \\
\hline Oligoryzomys flavescens & 21,3 & 2 & 6,9 & 3,96 \\
\hline DIDELPHIMORPHIA & & \\
\hline Didelphidae & & \multicolumn{5}{|l|}{} \\
\hline Thylamyini & 14,9 & 1 & 3,44 & 1,36 \\
\hline Thylamys pallidior & - & $\mathbf{2 9}$ & $\mathbf{1 0 0}$ & - \\
\hline Total & $\mathbf{1 0 8 8 , 5}$ & - & - & $\mathbf{1 0 0}$ \\
\hline Total Biomasa & $\mathbf{9}$ & - & - & - \\
\hline Ntaxa
\end{tabular}

Tabla 1. Composición taxonómica de presas contenidas en egagrópilas de Megascops choliba. Abreviaturas: Número Mínimo de Individuos (MNI, \%MNI); porcentaje de biomasa (\%B).
Con respecto a la biomasa, G. griseoflavus, la especie presa de mayor peso promedio detectada $(67,5 \mathrm{~g})$, fue la que representó el mayor aporte de biomasa de micromamíferos en la dieta de $M$. choliba $(31 \%)$. En menor medida, P. xanthopygus contribuyó en un $26,18 \%$, y C. musculinus -si bien fue la especie presa más frecuente-, aportó un 14,05\% de biomasa a la muestra (Tabla 1).

\section{Alteraciones tafonómicas}

\section{Representación anatómica}

Los valores calculados correspondientes a la representación anatómica se presentan en la Tabla 2. El MNE total fue de 1415. El promedio de abundancia relativa (Ri) fue de $56,32 \%$, donde

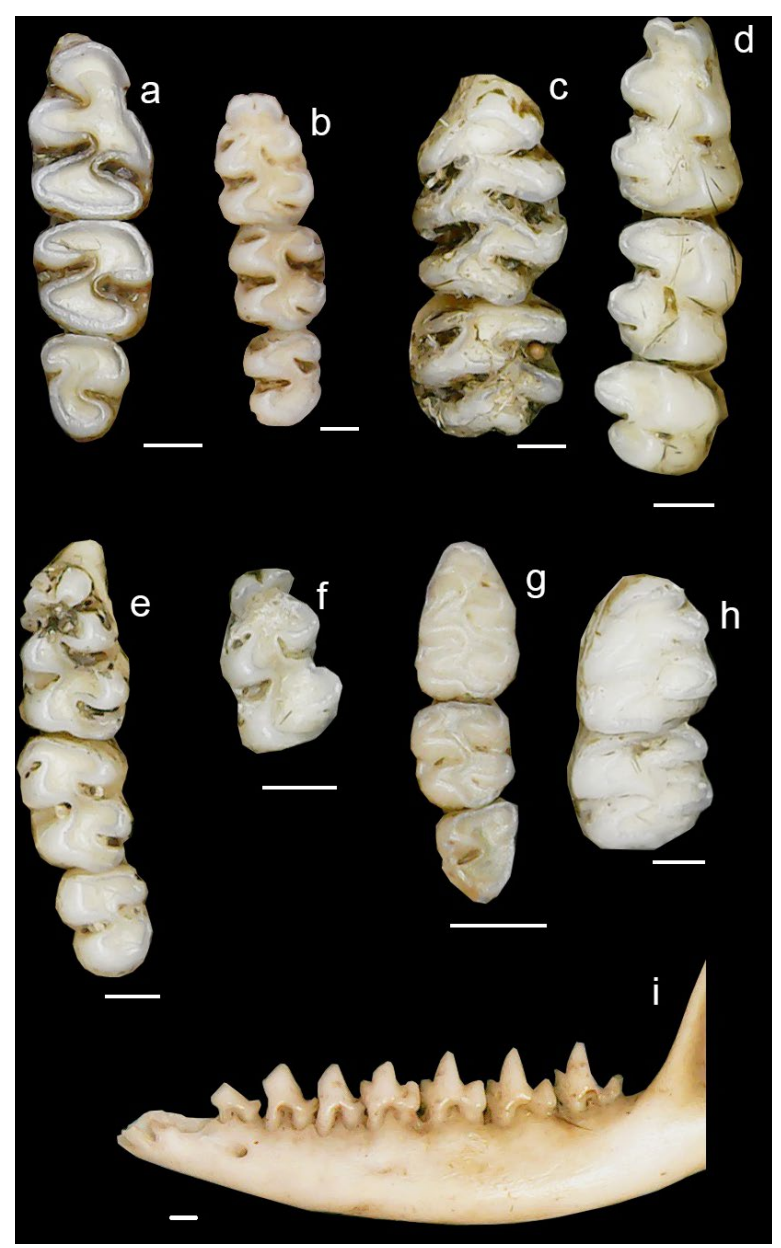

Figura 2. Series molares de micromamíferos presa identificados en egagrópilas de Megascops choliba. En vista oclusal: a- serie inferior derecha Phyllotis xanthopygus; bserie inferior izquierda Oligoryzomys flavescens; c- serie inferior izquierda Graomys griseoflavus; d- serie inferior derecha Oxymycterus rufus; e- serie inferior izquierda Akodon dolores; f- serie inferior izquierda Akodon polopi; gserie inferior izquierda Calomys musculinus; h- serie inferior izquierda Calomys venustus; i- en vista labial hemandíbula izquierda Thylamys pallidior. Escala $1 \mathrm{~mm}$. 
se destacan húmeros $(100 \%)$, pelvis $(100 \%)$, radios $(98,27 \%)$, tibias $(96,55 \%)$ y mandíbulas $(81,03 \%)$ (Tabla 2).

De acuerdo con los índices calculados para evaluar la relación de la representación entre partes esqueletarias, los resultados de los dos índices sobre la relación entre elementos poscraneales y craneales evidenciaron mejor preservación de los primeros con respecto a los segundos (índice $\mathrm{pc} / \mathrm{c}=408$, índice $\mathrm{h}+\mathrm{f} / \mathrm{md}+\mathrm{mx}=133$ ). Por su parte, la relación entre elementos distales y proximales del esqueleto (índice $t+u / f+h$ ) indica una proporción más equilibrada entre ambos conjuntos de elementos, dado que arroja un valor de 95,14\%. No obstante, se evidencia una leve pérdida de tibias y ulnas respecto de fémures y húmeros.

\begin{tabular}{|l|c|c|c|}
\hline Elemento anatómico & Ei & MNE & Ri \\
\hline Maxilar & 2 & 30 & 51,72 \\
\hline Mandíbula & 2 & 47 & 81,03 \\
\hline Escápula & 2 & 37 & 63,79 \\
\hline Húmero & 2 & 58 & 100 \\
\hline Radio & 2 & 57 & 98,27 \\
\hline Ulna & 2 & 42 & 72,41 \\
\hline Pelvis & 2 & 58 & 100 \\
\hline Fémur & 2 & 45 & 77,58 \\
\hline Tibia & 2 & 56 & 96,55 \\
\hline Vértebra & 55 & 479 & 30,03 \\
\hline Molares (aislados) & 12 & 24 & 3,44 \\
\hline Incisivos (aislados) & 4 & 14 & 24,13 \\
\hline Calcáneo & 2 & 31 & 53,44 \\
\hline Astrágalo & 2 & 9 & 15,51 \\
\hline Costilla & 24 & 254 & 18,24 \\
\hline Metapodio & 20 & 174 & 15 \\
\hline Total MNE & & $\mathbf{1 4 1 5}$ & - \\
\hline Promedio Ri & & - & $\mathbf{5 6 , 3 2}$ \\
\hline Poscráneo/Cráneo & & - & - \\
\hline$[(f+t+h+r+u) \times 8 /(m b+m x+m) \times 5] \times 100$ & & $\mathbf{4 0 8 \%}$ & - \\
\hline$[(h+f) /(m b+m x)] \times 100$ & & $\mathbf{1 3 3 \%}$ & - \\
\hline Elemento distal /proximal & & - & - \\
\hline$[(t+u) /(f+h)] \times 100$ & $\mathbf{9 5 , 1 4 \%}$ & - \\
\hline
\end{tabular}

Tabla 2. Porcentaje de abundancia relativa de partes esqueletales contenidas en egagrópilas de Megascops choliba. Abreviaturas: Mínimo Número de Elementos (MNE); Frecuencia del elemento en el esqueleto (Ei); Porcentaje de Abundancia Relativa (Ri). Los porcentajes de abundancia relativa se calcularon acordes con un $\mathrm{MNI}$ de 29. ${ }^{\text {a Se tomó }}$ un valor promedio, ya que varían en función de las distintas especies.

\section{Patrones de fractura}

Los grados y porcentajes de fracturas se presentan en la Tabla 3. Sobre esta variable, se registraron índices elevados de fracturación en cráneos y mayores grados de completitud en mandíbulas y elementos del poscráneo. Tan es así, que las categorías mayormente representadas en cráneos fueron las de ejemplares rotos con huesos frontales y región cigomática intactos (categoría $3=33,3 \%$ ) y los fragmentos de hemimaxilares, izquierdos o derechos (categoría $9=23,3 \%$ ).

En el caso de las mandíbulas, el $65,95 \%$ de los ejemplares se recuperaron completos (categoría 1), el $27,65 \%$ presentó su rama ascendente parcialmente fracturada (categoría 2) y el 6,4\% restante perdió la región posterior (categoría 3).

Los elementos poscraneales se recuperaron completos, en elevadas proporciones cada uno. En este sentido, solo el $1,55 \%$ se registró con algún tipo de fracturación. Los radios, ulnas y fémures no presentaron fracturas de ningún tipo. Tanto en húmeros como en tibias se registraron evidencias de fracturación en un 3,39\% y 5,36\%, respectivamente.

\section{Digestión}

Se registraron distintas categorías de corrosión digestiva, tanto en dientes como en elementos poscraneales (Tabla 4 y Figura 3). En todos los elementos analizados, la categoría leve fue la de mayor representación. En menores proporciones, se registró la categoría moderada, y en un solo caso (fémur) se observó corrosión digestiva fuerte.

Respecto de los valores obtenidos en dientes, alrededor del $44 \%$ de los incisivos indicó acción digestiva. En incisivos aislados, un $35,71 \%$ de los elementos se agruparon en la categoría leve y un $7,15 \%$ en la categoría moderada. En el caso de la digestión en incisivos in situ, el 36,37\% indicaba daños ligeros, y el 9,08\%, modificaciones moderadas.

En molares, se registró un 29\% con corrosión digestiva. Los elementos aislados se asocian en un $16,66 \%$ con la categoría leve y en un $4,16 \%$ con la categoría moderada. Por su parte, en los molares in situ, la proporción es de $26,83 \%$ y $11,38 \%$, respectivamente. Por otro lado, alrededor de un $15 \%$ de los elementos del poscráneo exhibieron modificaciones por acción digestiva. En húmeros, se registró un $8,77 \%$ con modificaciones leves y un 


\begin{tabular}{|c|c|c|}
\hline & MNE & $\%$ \\
\hline \multicolumn{3}{|l|}{ Fractura de cráneos } \\
\hline 1 Cráneo completo & - & - \\
\hline $\begin{array}{l}2 \text { Cráneo con huesos frontales y maxilar intactos, y al menos la } \\
\text { mitad de la bóveda craneal }\end{array}$ & 3 & 10 \\
\hline 3 Cráneo roto con huesos frontales y región cigomática intactos & 10 & 33,3 \\
\hline $\begin{array}{l}4 \text { Fragmento de maxilar sin apófisis cigomática. Premaxilar con o } \\
\text { sin incisivo }\end{array}$ & 4 & 13,3 \\
\hline 5 Premaxilar, con o sin incisivos & - & - \\
\hline 6 Maxilar sin proceso cigomático y premaxilar & - & - \\
\hline $\begin{array}{l}7 \text { Maxilar o premaxilar derecho o izquierdo, con o sin incisivo, } \\
\text { y nasal }\end{array}$ & 2 & 6,7 \\
\hline 8 Premaxilar derecho o izquierdo, con o sin incisivo, y nasal & 4 & 13,3 \\
\hline 9 Fragmento de maxilar derecho o izquierdo & 7 & 23,3 \\
\hline \multicolumn{3}{|l|}{ Fractura de mandíbulas } \\
\hline 1 Mandíbula completa & 31 & 65,95 \\
\hline 2 Mandíbula con rama ascendente rota & 13 & 27,65 \\
\hline 3 Mandíbula con pérdida de rama ascendente & 3 & 6,4 \\
\hline $\begin{array}{l}4 \text { Mandíbula con pérdida de rama ascendente y borde inferior } \\
\text { roto }\end{array}$ & - & - \\
\hline 5 Sínfisis con o sin incisivo & - & - \\
\hline \multicolumn{3}{|l|}{ Fractura de poscráneo } \\
\hline Húmero & 58 & 100 \\
\hline Completo & 57 & 96,61 \\
\hline Epífisis proximal & - & - \\
\hline Epífisis distal & 1 & 3,39 \\
\hline Diáfisis & - & - \\
\hline Radio & 57 & 100 \\
\hline Completo & 57 & 100 \\
\hline Epífisis proximal & - & - \\
\hline Epífisis distal & - & - \\
\hline Diáfisis & - & - \\
\hline Ulna & 42 & 100 \\
\hline Completo & 42 & 100 \\
\hline Epífisis proximal & - & - \\
\hline Epífisis distal & - & - \\
\hline Diáfisis & - & - \\
\hline Fémur & 45 & 100 \\
\hline Completo & 45 & 100 \\
\hline Epífisis proximal & - & - \\
\hline Epífisis distal & - & - \\
\hline Diáfisis & - & - \\
\hline Tibia & 56 & 100 \\
\hline Completo & 53 & 94,64 \\
\hline Epífisis proximal & 2 & 3,57 \\
\hline Epífisis distal & 1 & 1,8 \\
\hline Diáfisis & - & - \\
\hline
\end{tabular}

Tabla 3. Categorías y porcentuales de fracturas registradas en elementos craneales y poscraneales contenidos en egagrópilas de Megascops choliba en el valle de Ongamira, Córdoba (sensu Montalvo et al., 2020; Andrews, 1990).
$3,5 \%$ asignable a la categoría moderada. Para el caso de los fémures, se registró un $8,9 \%$ con alteraciones de tipo leve, un $6,66 \%$ con modificaciones moderadas y un solo elemento con daños de tipo fuerte $(2,22 \%)$.

\section{Megascops choliba versus otros depredadores}

Con el objetivo de explorar la ordenación de los valores obtenidos en un espacio multivariado, se realizó un análisis de componentes principales (PCA, por sus siglas en inglés), incluyendo datos obtenidos para aves rapaces Strigiformes (Tyto furcata, Athene cunicularia, Bubo virginianus, Pseudoscops clamator, Strix chacoensis), Accipitriformes (Geranoaetus melanoleucus, Geranoaetus polyosoma) y mamíferos carnívoros (Conepatus chinga, Lycalopex gymnocercus, Puma concolor).

El resultado observado en la Figura 4 indica que el componente 1 describe el $85 \%$ de la variación existente en la matriz de datos. Sumando el componente 2 se explica aproximadamente un $92 \%$ de la varianza en la muestra.

Los índices tafonómicos calculados indican, en el sector negativo del componente 1, una mayor correlación entre los valores calculados en este trabajo y los reportados para $S$. chacoensis en el Desierto de Monte Central (López, Fernández, Castillo y Pereyra-Lobos, 2018), Athene cunicularia (Montalvo et al., 
2020), Tyto furcata (Iglesias, 2009) y en menor medida, Bubo virginianus ñacurutu (Gómez, 2007).

\begin{tabular}{|l|c|c|c|c|c|}
\hline Elemento & \% Ausente & $\%$ Leve & \% Moderada & $\%$ Fuerte & $\%$ Extrema \\
Húmero & 87,71 & 8,77 & 3,5 & - & - \\
Fémur & 82,22 & 8,9 & 6,66 & 2,22 & - \\
Incisivo (aislado) & 57,14 & 35,71 & 7,15 & - & - \\
Incisivo in situ & 54,55 & 36,37 & 9,08 & - & - \\
Molar (aislado) & 79,16 & 16,66 & 4,16 & - & - \\
Molar in situ & 61,79 & 26,83 & 11,38 & - & - \\
\hline
\end{tabular}

Tabla 4. Grados y porcentajes de acción digestiva en especímenes óseos contenidos en egagrópilas de Megascops choliba en el valle de Ongamira, Córdoba (sensu Fernández et al., 2017; Andrews, 1990).

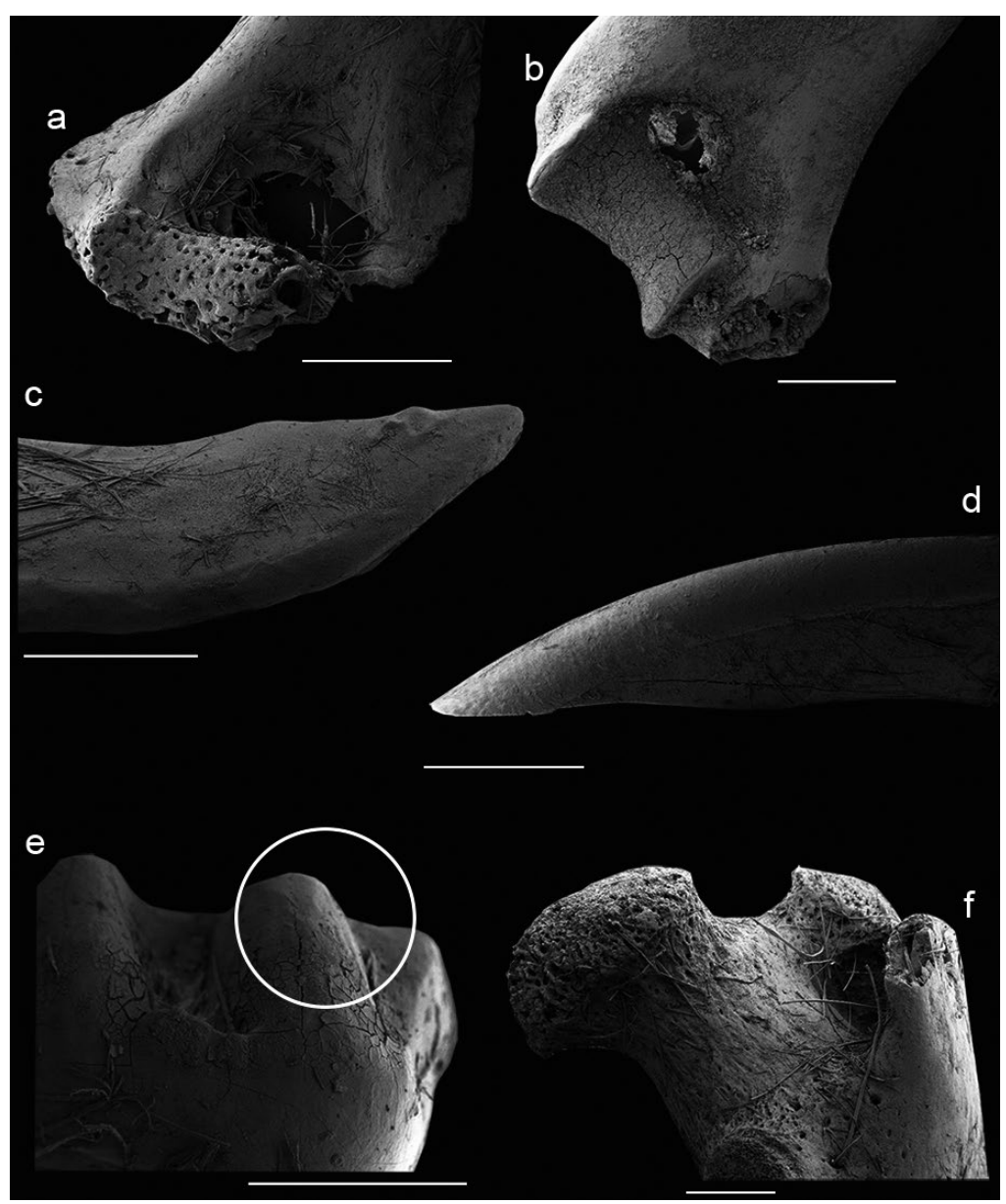

Figura 3. Elementos digeridos por Megascops choliba: a- digestión moderada sobre porción de húmero distal de roedor sigmodontino; b- porción distal de húmero de roedor sigmodontino con modificaciones leves; c- incisivo inferior en vista lateral de roedor sigmodontino con alteraciones leves; d-incisivo superior en vista lateral de roedor sigmodontino con modificaciones moderadas; e- molar inferior en vista lateral con modificaciones leves por digestión; f- porción proximal de fémur de sigmodontino con modificaciones de tipo fuerte. Escala $1 \mathrm{~mm}$.

\section{DISCUSIÓN}

\section{Megascops choliba como depredador multitaxa}

Se identificaron micromamíferos en un $40 \%$ de las egagrópilas de $M$. choliba. Los ítems presa registrados fueron ocho roedores sigmodontinos y 2018). También puede existir una variación en la abundancia de elementos esqueletales y grados de modificación por fractura y digestión, dados por factores ambientales y estacionales, por los tamaños y edades de los depredadores y presas y por el tipo de sitio de acumulación (e.g., perchas vs. nidos), entre otros (Andrews, 1990; Williams, 2001; López et al., 2017a). 


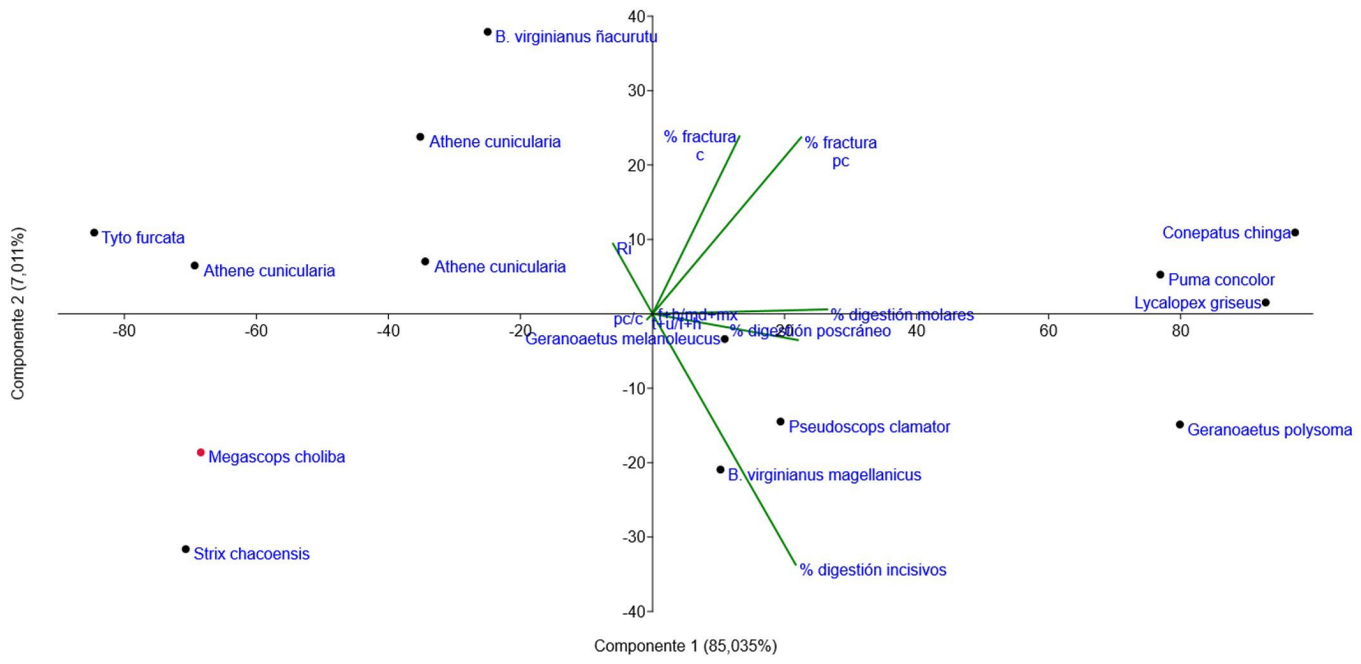

Figura 4. Análisis de componentes principales (basado en variables tafonómicas) de conjuntos acumulados en egagrópilas de Megascops choliba en comparación con otros valores generados por depredadores sudamericanos. Tyto furcata (Iglesias, 2009), Bubo virginianus ñacurutu (Gómez, 2007), Bubo virginianus magellanicus (Montalvo et al., 2016), Pseudoscops clamator (Rudzik, Fernández y Carrera, 2015), Strix chacoensis (López et al., 2018), Athene cunicularia (Montalvo et al., 2020), Geranoaetus melanoleucus, Geranoaetus polyosoma (López et al., 2017b), Lycalopex gymnocercus (Gómez y Kaufmann, 2007), Puma concolor (Montalvo et al., 2007) y Conepatus chinga (Montalvo, Pessino y Bagatto, 2008).

En términos ecológicos, el taxón de mayor representación en egagrópilas de $M$. choliba fue $C$. musculinus, un filotino típico de agroecosistemas y pastizales (Patton et al., 2015). El resto de las especies determinadas representan elementos típicos de pastizales de altura, arbustos, sustratos rocosos (e.g., Akodon spp. P. xanthopygus y O. rufus) y áreas de bosque de mayor y menor densidad (G. griseoflavus y T. pallidior) (Albanese, 2011; Patton et al., 2015).

Estas observaciones indicaron que $M$. choliba presentaría conductas de caza vinculadas a presas con hábitos principalmente cursoriales y nocturnos. Al respecto, Motta-Junior (2002) indicó la presencia de Necromys lasiurus, Calomys tener y Oligorizomys nigripes en muestras de la misma rapaz del sudeste de Brasil. Todos ellos son taxones de hábitos preferentemente nocturnos y crepusculares.

\section{Patrones tafonómicos de huesos y dientes digeridos por Megascops choliba}

Respecto de los patrones tafonómicos registrados, se observaron similitudes y diferencias en relación con estudios previos realizados sobre acumulaciones de otras Strigiformes diurnas y nocturnas (Montalvo y Fernández, 2019). En cuanto a los valores de representación anatómica obtenidos, el porcentaje de abundancia relativa de partes esqueletales fue ligeramente superior a los reportados para otras Strigiformes, lo cual indica una buena preservación de todos los elementos anatómicos del esqueleto en las egagrópilas analizadas. Valores similares se obtuvieron en muestras de $T$. furcata (Iglesias, 2009) y $B$. v. ñacurutu (Gómez, 2007). No obstante, los índices calculados sobre la proporción de elementos esqueléticos de estos dos depredadores indican menor preservación de elementos poscraneales por sobre los cráneo-mandibulares que los revelados para M. choliba. En este sentido, Montalvo et al. (2020) y López et al. (2018) reportaron valores de preservación equitativos en muestras de $A$. $\mathrm{Cu}$ nicularia y $S$. chacoensis, respectivamente.

Con respecto a la representación de elementos distales y proximales, se observó equitatividad entre ambos elementos. Al respecto, se alcanzaron valores similares en restos acumulados por $B$. $v$. magellanicus y $S$. chacoensis (Montalvo, Fernández y Tallade, 2016; López et al., 2018).

Las observaciones sobre fracturas en la totalidad de restos cráneo-mandibulares indicaron una elevada proporción de los elementos con algún tipo de rotura. En cráneos, los porcentajes de fragmentación fueron elevados; se observó un mayor nivel de completitud en mandíbulas y modificaciones mínimas en restos poscraneales.

Los valores obtenidos sobre fracturas en restos cráneo-mandibulares fueron mayores a los calculados sobre otras rapaces Strigiformes (e.g. T. furcata y $S$. chacoensis), aunque revelaron similitudes con los reportados para A. cunicularia (Iglesias, 2009; López et al., 2018; Montalvo et al., 2020). Esto resulta interesante ya que, si bien se observó un alto porcentaje de fracturas en restos cráneo-mandibulares, el valor de abundancia relativa de partes esqueletales indicó menor pérdida de huesos que otras Strigiformes (Montalvo y Fernández, 2019). Por otro lado, el porcentaje de fracturas en elementos del poscráneo indicó mejores condiciones de 
preservación que otras rapaces estudiadas hasta el momento, incluso aquellas de modificación mínima, como T. furcata (Montalvo y Fernández, 2019).

Se detectó una moderada frecuencia de restos con acción digestiva. Respecto de los distintos grados de digestión sobre dientes, la categoría leve de modificación fue la de mayor representación y, en menores proporciones, las categorías moderada y fuerte. Las proporciones obtenidas fueron más elevadas que las reportadas en muestras de T. furcata y B. v. ñacurutú, y se relevaron porcentuales similares en restos de $A$. cunicularia (Gómez, 2007; Iglesias, 2009; Montalvo et al., 2020). Por su parte, en restos acumulados por $S$. chacoensis, los valores fueron más elevados en incisivos (ca. 50\%), aunque la digestión en molares no alcanzó el 10\% (López et al., 2018). En cuanto a los elementos del poscráneo, una baja proporción de los huesos exhiben modificaciones por acción digestiva. Estos resultados revelarían que $M$. choliba modifica estos elementos anatómicos en menor proporción que la mayoría de otras rapaces Strigiformes sudamericanas conocidas. Tan es así que, en muestras de T. furcata, una rapaz de modificación mínima, los valores de digestión en elementos del poscráneo fueron mayores que los alcanzados en este trabajo (Iglesias, 2009; Montalvo y Fernández, 2019; López, 2020).

Estas evaluaciones resultan interesantes, ya que la digestión es la variable más confiable a la hora de realizar inferencias sobre agentes acumuladores en asociaciones arqueológicas y fósiles (Andrews, 1990; Fernández et al., 2017). Esto se debe a que otros patrones tafonómicos (e.g., abundancia relativa y fracturación) resultan propensos a la equifinalidad a causa de la acción de distintos procesos tafonómicos (e.g., abrasión o pisoteo).

En resumen, las observaciones de huesos contenidos en sus egagrópilas indicaron una elevada preservación de elementos, similar a T. furcata. concentraron principalmente en la categoría ligera y, en menor proporción, en las categorías moderada y fuerte. No se registró acción digestiva de tipo extrema (Tabla 4).

Las evaluaciones tafonómicas detectadas indicaron patrones similares respecto de otras rapaces Strigiformes, con mayor afinidad con $S$. chacoensis y A. cunicularia (Figura 4). Si bien es posible su ocurrencia, ambas rapaces no han sido registradas en el sector bajo estudio hasta el momento (Mignino et al., 2018).

En función de lo expuesto, y siguiendo los lineamientos de Andrews (1990), la sumatoria y categorización de los atributos tafonómicos revela que M. choliba podría ubicarse entre las categorías 2 y 3 de modificación (Tabla 5).

\section{CONCLUSIONES}

\section{Contribuciones para estudios arqueológicos y paleontológicos}

Este trabajo constituye el primer estudio tafonómico de restos óseos y dentarios de micromamíferos recuperados en egagrópilas generadas por M. choliba. El registro de su ocurrencia en aleros, paredones, roquedales y áreas de bosque al aire libre, donde se ha documentado la presencia de ocupaciones humanas, exige la necesidad de evaluar su función como agente acumulador de restos de pequeños mamíferos.

Este punto es clave ya que la identificación certera de los agentes causantes de la acumulación permitirán reducir sesgos y posibles interpretaciones erróneas sobre los conjuntos (Andrews, 1990).

A la vez, si bien es posible estimar la acción acumuladora de determinados agentes -humanos o no humanos-, es factible que esas asociaciones Los grados de rotura fueron muy bajos en mandíbulas y elementos poscraneales, mientras que en cráneos alcanzaron a la totalidad de los ejemplares (ver Tabla 3). Finalmente, las modificaciones ocasionadas por digestión gástrica se

\begin{tabular}{|l|l|l|l|l|l|}
\hline Variable tafonómica & Categoría 1 & Categoría 2 & Categoría 3 & Categoría 4 & Categoría 5 \\
\hline Abundancia relativa & & & & & \\
\hline Poscráneo/cráneo & & & & & \\
\hline Distal/proximal & & & & & \\
\hline Fracturas/maxilar & & & & \\
\hline Fractura/mandíbula & & & & & \\
\hline Fractura/poscráneo & & & & & \\
\hline Digestión/incisivos & & & & \\
\hline Digestión/molares & & & & & \\
\hline Digestión/poscráneo & & & & & \\
\hline
\end{tabular}

Tabla 5. Categorización de modificaciones en huesos de micromamíferos contenidos en egagrópilas de Megascops choliba de acuerdo con las variables tafonómicas analizadas (sensu Andrews, 1990). 
no posean un origen único y común; sino más bien que revistan una naturaleza mixta (Montalvo et al., 2017). Lo expuesto aquí indica la importancia de conocer de antemano las posibles variables involucradas en el origen de las asociaciones faunísticas.

Esta clase de estudios, además, se constituyen como un requisito fundamental a la hora de evaluar la ocurrencia de pequeños mamíferos y sus implicancias paleoecológicas. En este sentido, la información taxonómica presentada incrementa el conocimiento sobre comunidades actuales de micromamíferos en el Bosque Chaqueño Serrano de la provincia de Córdoba, hecho que contribuye al fortalecimiento de las reconstrucciones e inferencias paleoambientales en estudios arqueológicos y paleontológicos.

\section{Agradecimientos}

A los habitantes del valle (Mónica, Miguel, Carola y Feliciano Supaga, Vilma y Gustavo Roca y Alfredo Castillo). A Pablo Eguia, por su aporte y autorización para el uso de imagen de Megascops choliba. Las editoras y los revisores mejoraron considerablemente la calidad de este trabajo. Manuel López realizó una lectura crítica y pormenorizada del manuscrito original. Camila Brizuela, Andrés Robledo y Paula Weihmüller colaboraron con las tareas de campo. Finalmente, a quienes apoyan desde la formación y financiación para lograr objetivos propuestos; PICT 2016-0264 (directora Roxana Cattáneo) PIP CONICET 11220130100137CO (director Andrés Izeta).

\section{REFERENCIAS CITADAS}

Albanese, M. S. (2011). Ecología de la marmosa pálida Thylamys pallidior (Marsupialia, Didelphidae), en el desierto de Monte Central. Mastozoología Neotropical, 18, 147-157.

Alcaráz, A. P. (2020). El conjunto de fauna menor del sitio La Modesta: un aporte al estudio de la subsistencia durante el Holoceno medio en la transición pampeanopatagónica oriental (Buenos Aires, Argentina).

Arqueología, 26, 103-126.

Andrews, P. (1990). Owls, Caves and Fossils. Predation, Preservation, and Accumulation of Small Mammal Bones in Caves, with the Analysis of the Pleistocene Cave Faunas from Westbury-sub-mendip, Somerset. UK. Natural History Museum Publications.
Behrensmeyer, A. K. y Kidwell, S. M. (1985). Taphonomy's contributions to paleobiology. Paleobiology, 11, 105-119.

Borrero, L. A. (1990). Taphonomy of guanaco bones in Tierra del Fuego. Quaternary Research, 34, 361-371.

Cattáneo, G. R. e Izeta, A. D. (Eds.) (2016). Arqueología en el Valle de Ongamira, Córdoba (2010-2015). IDACOR-Museo de Antropología, Universidad Nacional de Córdoba. https://suquia.ffyh.unc.edu.ar/bitstream/ handle/suquia/639/Cattaneo_e_Izeta_2016_Cap03-1. pdf? sequence $=1$ \&isAllowed $=y$

Delgado, C. A. (2007). La dieta del Currucutú Megascops choliba (Strigidae) en la ciudad de Medellín, Colombia. SAO, 17, 111-114.

del Hoyo, J., Elliot, A. y Sargatal, J. (1999). Handbook of the birds of the World. Barn owls to hummingbirds. Vol 5. Lynx Edicions.

Fernández, F. J., Ballejo, F., Moreira, G., Tonni, E. y De Santis, L. J. M. (2011). Roedores cricétidos de la provincia de Mendoza. Guía cráneo-dentaria orientada para su aplicación en estudios zooarqueológicos. Sociedad Argentina de Antropología y Universitas Sarmiento.

Fernández, F. J., Teta, P., Barberena, R. y Pardiñas, U. F. J. (2012). Small mammal remains from Cueva Huenul 1, northern Patagonia, Argentina: Taphonomy and paleoenvironments since the Late Pleistocene. Quaternary International, 278, 22-31.

Fernández, F. J., Montalvo, C. I., Fernandez-Jalvo, Y., Andrews, P. y López, J. M. (2017). A reevaluation of the taphonomic methodology for the study of small mammal fossil assemblages of South America. Quaternary Science Reviews, 155, 37-49. http://dx. doi. org/10.1016/j.quascirev.2016.11.005

Fernández-Jalvo, Y., y Andrews, P. (1992). Small mammal taphonomy of Gran Dolina, Atapuerca (Burgos), Spain. Journal of Archaeological Science, 19, 407-428.

Frontini, R. y Escosteguy, P. (2015). El rol de los pequeños animales en los estudios arqueofaunísticos de Argentina. Archaeofauna, 24, 67-85.

Gómez, G. N. (2007). Predators categorization based on taphonomic analysis of micromammal's bone: a comparison to proposed models. En M. A. Gutiérrez, L. Miotti, G. Barrientos, G. Mengoni Goñalons y M. Salemne (Eds.), Taphonomy and Zooarchaeology in Argentina (pp. 1-16). BAR International series 1601. Archaeopress.

Gómez, G. N. y Kaufmann, C. A. (2007). Taphonomic analysis of Pseudalopex griseus (Grey, 1837). Scat assemblages and their archaeological implications. Journal of Taphonomy, 5, 59-70. 
Gutiérrez, M. A. (2009). Tafonomía: ¿Tiranía o multivocalidad? En R. Barberena, K. Borrazzo y L. A. Borrero (Eds.), Perspectivas actuales en arqueología argentina (pp. 55-88). Dunken.

Hammer, Ø., Harper, D. A. T. y Ryan, P. D. (2001). PAST: Paleontological statistics software package for education and data analysis. Palaeontologia Electronica, 4 (1), 9. http://palaeo-electronica.org/2001_1/past/issue1_01.htm.

Hilty, S. L. y Brown, L. (1996). A guide to the Birds of Colombia. Princeton University Press.

Iglesias, A. C. (2009). Tafonomía de pequeños vertebrados depredados por Buteo polyosoma (Aves, Falconiformes) (Tesis de grado inédita). Facultad de Ciencias Exactas y Naturales, Universidad Nacional de La Pampa, Santa Rosa, La Pampa, Argentina.

Izeta, A. (2005). South American camelid bone estructural density: what are we measuring? Comments on data sets, values, their interpretation and application. Journal of Archaeological Science, 32, 1159-1168. 10.1016/j.jas.2005.02.013

König, C. y Weick, F. (2010). Owls of the world. A\&C Black Publishers.

López, J. M. (2020). Actualistic taphonomy of barn owl pellet-derived small mammal bone accumulations in arid environments of South America. Journal of Quaternary Science, 35, 1057-1069. 10.1002/jqs.3251

López, J. M. y Chiavazza, H. (2020). Micromamíferos recuperados en sitios arqueológicos de precordillera en los Andes del Centro-Occidente Argentino (Holoceno Tardío): aspectos tafonómicos y paleoambientales. Latin American Antiquity, 31, 409-429.

López, J. M., Rosi, M., Tabeni, S., Bender, J. y Chiavazza, H. (2017a). Taphonomic analysis of small mammal remains preyed upon by wildcats (Carnivora: Felidae) from the central Monte Desert (Mendoza, Argentina). Boreas, 46, 282-293.

López, J. M., Fernández, F. J., Montalvo, C., Chiavazza, H. y De Santis, N. (2017b). The role of the Accipitriforms Geranoaetus melanoleucus and Geranoaetus polyosoma as small mammals bones acumulator in modern and archaeological sites from central western Argentina. Journal of Taphonomy, 15, 91-108.

López, J. M., Fernández, F. J., Castillo, L. y PereyraLobos, R. (2018). Taphonomy of small mammal bone acumulations generated by the Chaco Owl (Strix chacoensis, Strigiformes): a contribution to South American archaeological and palaeontological studies. Boreas, 47, 780-791.

Marti, C. D., Bechard, M. y Jaksic, F. M. (2007). Food habits. En D. M. Bird y K. L. Bildstein (Eds.), Raptor
Research and Management Techniques (pp. 129-152). Hancock House.

Mignino, J., Izeta, A. y Cattáneo, R. (2018). Modern and archaeological owl pellets as paleoenvironmental and taphonomic markers in human occupation contexts in the Ongamira Valley, Córdoba, Argentina. Journal of Archaeological Science: Reports, 18, 65-77. 10.1016/j.jasrep.2017.12.054

Montalvo, C. (2004). Paleobiología de la asociación faunística de Caleufú [La Pampa, Formación Cerro Azul, Mioceno Superior - Plioceno Inferior], a través de análisis tafonómicos (Tesis doctoral inédita). Universidad Nacional de La Plata, Facultad de Ciencias Exactas, La Plata, Buenos Aires, Argentina.

Montalvo, C., Pessino, M. y Bagatto, F. (2008). Taphonomy of the bones of rodent consumed by Andean hog-nosed skunks (Conepatus chinga, Carnívora, Mephitidae) in central Argentina. Journal of Archaeological Science, 35, 1481-1488.

Montalvo, C., Fernández F. J. y Tallade, P. (2016). The role of Bubo virginianus magellanicus as rodent bone accumulator in archaeological sites: a case study for the Atuel River (Mendoza, Argentina). International Journal of Osteoarchaeology 26(6):974-986. 10.1002/oa.2509

Montalvo, C. y Fernández F. J. (2019). Review of the actualistic taphonomy of small mammals ingested by South American predators. Its importance in the interpretation of the fossil record. Publicación Electrónica de la Asociación Paleontológica Argentina, 19, 18-46.

Montalvo, C., Fernández F. J., Bargo, S. M., Tomassini, R., Mehl, A. (2017). First record of a Late Holocene fauna associated with an ephemeral fluvial sequence in La Pampa Province, Argentina. Taphonomy and paleoenvironment. Journal of South American Earth Sciences, 76, 225-237.

Montalvo, C., Fernández F. J., Tomassini, R., Mignino, J., Kin, M. y Santillán, M. A. (2020). Spatial and temporal taphonomic study of bone accumulations of the burrowing owl (Athene cunicularia) in central Argentina. Journal of Archaeological Science: Reports, 30, 102-197. 10.1016/j.jasrep.2020.102197

Motta-Junior, J. C. (2002). Diet of breeding tropical Screech-owls (Otus choliba) in southeastern Brazil. Journal of Raptor Research, 36, 332-334.

Pardiñas, U. F. J. (1999). Tafonomía de microvertebrados en yacimientos arqueológicos de Patagonia (Argentina). Arqueología, 9, 265-340. 
Patton, J. L., Pardiñas, U. F. J. y D’Elia, G. (2015). Mammals of South America. Volume 2-Rodents. The University of Chicago Press.

Piovano E. L. D., Ariztegui, F., Cordoba, M., Cioccale, F. y Sylvestre, F. (2009). Hydrological Variability in South America Below the Tropic of Capricorn (Pampas and Patagonia, Argentina) During the Last 13.0 Ka. En F. Vimeux et al. (Eds.), Past Climate Variability in South America and Surrounding Regions. Developments in Paleoenvironmental Research, 14: 323-351.

Robledo, A. (2019). Arqueología en el valle de Ongamira (Deptos. de Ischilín y Totoral, Córdoba, Argentina). Paisajes y lugares de sociedades cazadoras recolectoras holocénicas (Tesis doctoral). Facultad de Filosofía y Humanidades, Universidad Nacional de Córdoba, Córdoba, Argentina.

Rudzik, S. M., Fernández, F. J. y Carrera, J. D. (2015). Taphonomic analysis of micromammal remains from striped owl (Pseudoscops clamator) in Northeastern Buenos Aires Province, Argentina: implications for archaeological sites formation. International Journal of Osteoarchaeology, 25, 550-563.

Saavedra, B. y Simonetti, J. A. (1998). Small mammals taphonomy: Intraspecific bone assemblage comparison between South and North American Barn Owl, Tyto alba, populations. Journal of Archaeological Science, 25, 165-170.

Scheifler, N. Teta, P. y Pardiñas, U. F. J. (2012). Small mammals (Didelphimorphia and Rodentia) of the archaeological site Calera (Pampean Region, Buenos
Aires province, Argentina): Taphonomic history and Late Holocene environments. Quaternary International, 278, 32-44.

Scheifler, N., Massigoge, A., Kaufmann, C., Rafuse, D., González, M., Gutiérrez, M. y Álvarez, M. C. (2020). Modern bone distribution in the Pampas of Argentina: taphonomic implications for the regional archaeological record. En S. Martínez, A. Rojas y F. Cabrera (Eds.), Actualistic Taphonomy in South América. Topics in Geobiology 48. Springer. 10.1007/978-3-030-20625-3_11

Teta, P., Medina, M., Pastor, S., Rivero, D. y Paradela, H. (2005). Holochilus brasiliensis (Rodentia, Cricetidae) en conjuntos arqueofaunísticos del Holoceno Tardío de la provincial de Córdoba (Argentina). Mastozoología Neotropical, 12, 271-275.

Williams, J. P. (2001). Small mammal deposits in archaeology: a taphonomic investigation of Tyto alba (barn owl) nesting and roosting sites (PhD thesis). University of Sheffield.

Yanes, Y., Izeta, A. D., Cattáneo, R., Costa, T. y Gordillo, S. (2014). Holocene (4.5-1.7 cal. Kyr BP) Paleoenvironmental conditions inferred from entire-shell andintra-shell stable isotope composition of terrestrial gastropods. The Holocene, 180(1), 135-144.

Zárate, M. (2016). Explorando la historia geológica del Alero Deodoro Roca. En R. Cattáneo y A. D. Izeta (Eds.), Arqueología del Valle de Ongamira, 2010-2015 (pp. 43-56). Universidad Nacional de Córdoba. 
